\title{
Limits on muon-neutrino to tau-neutrino oscillations induced by a sterile neutrino state obtained by OPERA at the CNGS beam
}

\section{The OPERA collaboration}

E-mail: alessandro.paoloni@lnf.infn.it, alessandra.pastore@ba.infn.it

Abstract: The OPERA experiment, exposed to the CERN to Gran Sasso $\nu_{\mu}$ beam, collected data from 2008 to 2012 . Four oscillated $\nu_{\tau}$ Charged Current interaction candidates have been detected in appearance mode, which are consistent with $\nu_{\mu} \rightarrow \nu_{\tau}$ oscillations at the atmospheric $\Delta m^{2}$ within the "standard" three-neutrino framework. In this paper, the OPERA $\nu_{\tau}$ appearance results are used to derive limits on the mixing parameters of a massive sterile neutrino.

Keywords: Oscillation, Neutrino Detectors and Telescopes, Beyond Standard Model ARXIV EPRINT: 1503.01876 


\section{Contents}

1 Introduction 1

2 Detector, beam, and data sample $\quad 2$

3 Search for $\nu_{\tau}$ interactions $\quad 2$

4 Sterile neutrino search via $\nu_{\mu} \rightarrow \nu_{\tau}$ oscillations $\quad 3$

$\begin{array}{lll}5 & \text { Conclusions } & 7\end{array}$

$\begin{array}{ll}\text { The OPERA collaboration } & 11\end{array}$

\section{Introduction}

The OPERA experiment [1] operated in the CERN Neutrinos to Gran Sasso (CNGS) beam produced at CERN and directed towards the Gran Sasso Underground Laboratory of INFN (LNGS), $730 \mathrm{~km}$ away, where the detector is located. The experiment is unique in its capability to observe $\nu_{\tau}$ appearance on an event-by-event basis. Nuclear emulsion films instrumenting the target allow the detection of the short-lived $\tau$ lepton decay, and hence the identification of $\nu_{\tau}$ Charged Current (CC) interactions. The standard three-neutrino oscillation framework predicts $\nu_{\mu} \rightarrow \nu_{\tau}$ oscillations with close-to-maximal mixing at the so-called atmospheric scale, $\Delta m_{32}^{2} \sim 2.4 \times 10^{-3} \mathrm{eV}^{2}$ [2], i.e. in the oscillation parameters region discovered by detecting atmospheric neutrinos [3]. OPERA has observed four $\nu_{\tau}$ $\mathrm{CC}$ interaction candidate events [4-7], consistent with the expectation of the standard oscillation framework at this scale. This result represents the first direct evidence of $\nu_{\mu} \rightarrow \nu_{\tau}$ oscillation in appearance mode.

In the present paper, limits are derived on the existence of a massive sterile neutrino. The excess of $\nu_{e}\left(\bar{\nu}_{e}\right)$ observed by the LSND [8] and MiniBooNE [9] collaborations and the so-called reactor [10] and Gallium [11, 12] neutrino anomalies are also interpreted as due to the existence of a fourth sterile neutrino with mass at the $\mathrm{eV}$ scale. In relation to this issue, it is worth mentioning that the effective number of neutrino-like species decoupled from the primeval plasma measured by the Planck collaboration is $3.15 \pm 0.23$ at $95 \%$ Confidence Level (CL) [13].

Neutrino oscillations at large $\Delta m^{2}$ have been searched for by several short baseline experiments. The most stringent limits on $\nu_{\mu} \rightarrow \nu_{\tau}$ oscillations were set by the NOMAD [14] and CHORUS [15] experiments, with high sensitivity for $\Delta m^{2}$ values larger than $10 \mathrm{eV}^{2}$.

In the following, a short description of the OPERA experimental setup and of the procedure used to detect $\nu_{\tau}$ interactions is given, the data analysis is described and exclusion regions in the parameter space are derived. 


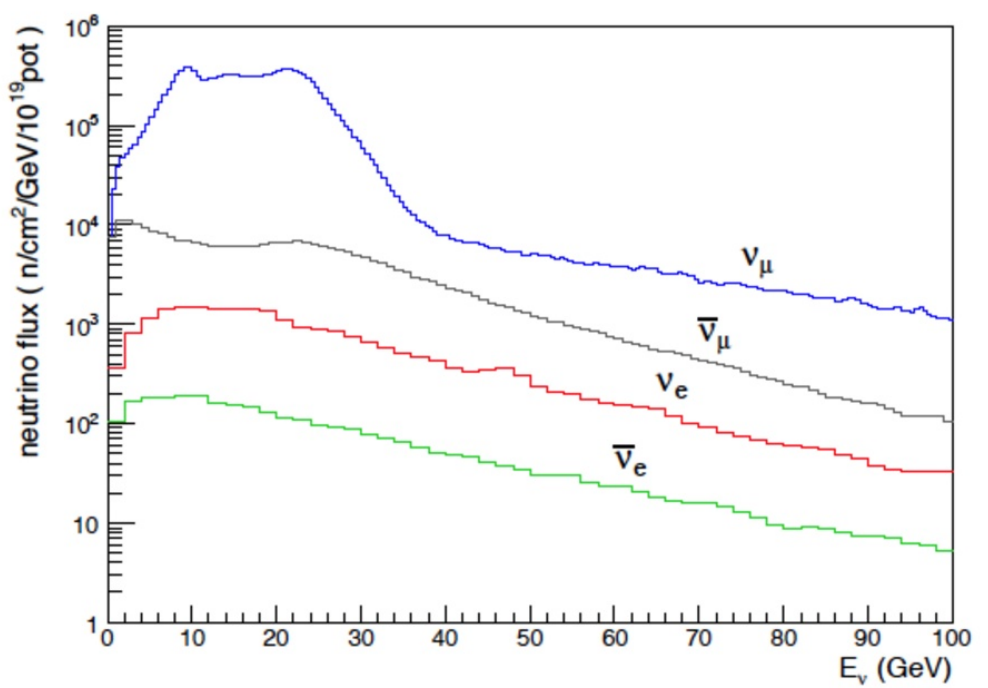

Figure 1. Fluxes of the different CNGS beam neutrino components at LNGS.

\section{Detector, beam, and data sample}

In OPERA, CNGS neutrinos interacted in a massive target made of lead plates interspaced with nuclear emulsion films acting as high accuracy tracking devices [16]. This kind of detector is historically called Emulsion Cloud Chamber (ECC). The OPERA detector is made of two identical Super Modules, each consisting of a target section and a muon magnetic spectrometer. Each target section has a mass of about 625 tons and is made of $\sim 75000$ units called bricks. A target brick consists of 56 lead plates $1 \mathrm{~mm}$-thick interleaved with 57 nuclear emulsion films $300 \mu$ m-thick for a mass of $8.3 \mathrm{~kg}$. Its total thickness along the beam direction corresponds to about 10 radiation lengths. The bricks are assembled in vertical walls instrumented with scintillator strips (Target Tracker detectors, TT) to trigger the read-out and locate neutrino interactions within the target.

OPERA was exposed to the CNGS $\nu_{\mu}$ beam generated by protons from the SPS accelerator at CERN $[17,18]$. The contaminations of $\bar{\nu}_{\mu}, \nu_{e}$ and $\bar{\nu}_{e}$ CC interactions at LNGS, relative to $\nu_{\mu} \mathrm{CC}$ interactions, are $2.1 \%, 0.9 \%$ and less than $0.1 \%$, respectively. The contamination of prompt $\nu_{\tau}$ is negligible. The average $\nu_{\mu}$ energy is $17 \mathrm{GeV}$. The energy spectra of the four beam components at the detector site are shown in figure 1 [19].

The data taking, based on a minimum bias interaction trigger from the TT scintillators, started in 2008 and ended in December 2012. OPERA collected data corresponding to $17.97 \times 10^{19}$ protons on target (pot) with 19505 recorded events. The data sample used in this analysis is defined following the selection criteria described in [5] and corresponds to about $75 \%$ of the total statistics.

\section{Search for $\nu_{\tau}$ interactions}

Bricks selected as candidates to contain CNGS neutrino interactions are analysed following the procedure described in detail in [5]. Here we just recall the main steps of the analysis. 
The brick where a neutrino interaction occurred is predicted by the electronic detectors and extracted from the target by an automatic brick manipulator system. Two extra low background emulsion films (Changeable Sheets, CS) [20] located downstream of the brick act as an interface between the brick and the electronic detectors. If the measurement of the CS yields tracks related to the neutrino interaction, the emulsion films of the brick are developed. Their analysis provides the three dimensional reconstruction of the neutrino interaction and of the possible decay vertices of short-lived particles [21] with micrometric accuracy.

This procedure has led to the detection of four $\nu_{\tau}$ CC interaction candidates. The total expected background in the analysed sample amounts to $0.23 \pm 0.05$ events. The absence of a $\nu_{\mu} \rightarrow \nu_{\tau}$ oscillation signal, i.e. the hypothesis of the four events being background, is excluded with a significance of $4.2 \sigma$ [7].

\section{Sterile neutrino search via $\nu_{\mu} \rightarrow \nu_{\tau}$ oscillations}

In [7] the detection of four $\nu_{\tau}$ CC events is compared to the expectation for $\nu_{\mu} \rightarrow \nu_{\tau}$ oscillations in the atmospheric sector, computed within a simplified two-flavour scheme assuming full mixing and $\left|\Delta m_{32}^{2}\right|=2.32 \times 10^{-3} \mathrm{eV}^{2}$ [22]. The expected number of events is $2.30 \pm 0.46(2.21 \pm 0.44)$ assuming normal (inverted) hierarchy of neutrino masses; the number is obtained by rescaling the value given in [7] for $\left|\Delta m_{32}^{2}\right| \approx\left|\Delta m_{31}^{2}\right| \approx\left|\Delta m^{2}\right|=$ $2.43 \times 10^{-3} \mathrm{eV}^{2}\left(\left|\Delta m^{2}\right|=2.38 \times 10^{-3} \mathrm{eV}^{2}\right)[2]$, where $\Delta m^{2}$ is defined as $m_{3}^{2}-\frac{\left(m_{1}^{2}+m_{2}^{2}\right)}{2}$. By including the background, $2.53 \pm 0.46(2.44 \pm 0.44)$ events are expected in total. The error, which is dominated by the uncertainty on the $\tau$ detection efficiency and on the $\nu_{\tau}$ interaction cross section, also takes into account the experimental precision on the atmospheric oscillation parameters. The observation of four events is compatible with these expectations. Despite the limited statistics, an excess or a deficit of $\nu_{\tau}$ interactions due to $\nu_{\mu} \rightarrow \nu_{\tau}$ oscillations induced by the mixing with a sterile neutrino can be evaluated.

In presence of a fourth sterile neutrino with mass $m_{4}$, the oscillation probability is a function of the $4 \times 4$ mixing matrix $U$ and of the three squared mass differences. Defining $C=2\left|U_{\mu 3}\right|\left|U_{\tau 3}\right|, \Delta_{i j}=1.27 \Delta m_{i j}^{2} L / E(i, j=1,2,3,4), \phi_{\mu \tau}=\operatorname{Arg}\left(U_{\mu 3} U_{\tau 3}^{*} U_{\mu 4}^{*} U_{\tau 4}\right)$ and $\sin 2 \theta_{\mu \tau}=2\left|U_{\mu 4}\right|\left|U_{\tau 4}\right|$, the $\nu_{\mu} \rightarrow \nu_{\tau}$ oscillation probability $P(E)$ can be parametrised as:

$$
\begin{aligned}
P(E)= & C^{2} \sin ^{2} \Delta_{31}+\sin ^{2} 2 \theta_{\mu \tau} \sin ^{2} \Delta_{41} \\
& +\frac{1}{2} C \sin 2 \theta_{\mu \tau} \cos \phi_{\mu \tau} \sin 2 \Delta_{31} \sin 2 \Delta_{41} \\
& -C \sin 2 \theta_{\mu \tau} \sin \phi_{\mu \tau} \sin ^{2} \Delta_{31} \sin 2 \Delta_{41} \\
& +2 C \sin 2 \theta_{\mu \tau} \cos \phi_{\mu \tau} \sin ^{2} \Delta_{31} \sin ^{2} \Delta_{41} \\
& +C \sin 2 \theta_{\mu \tau} \sin \phi_{\mu \tau} \sin 2 \Delta_{31} \sin ^{2} \Delta_{41}
\end{aligned}
$$

where $\Delta m_{31}^{2}$ and $\Delta m_{41}^{2}$ are expressed in $\mathrm{eV}^{2}, L$ in $\mathrm{km}$ and $E$ in $\mathrm{GeV}$. Given the long baseline and the average CNGS neutrino energy, $P(E)$ is independent of $\Delta m_{21}^{2}$, since $\Delta_{21} \approx 4 \times 10^{-3}$. The terms proportional to $\sin \phi_{\mu \tau}$ are CP-violating, while those proportional to $\sin 2 \Delta_{31}$ are sensitive to the mass hierarchy of the three standard neutrinos, normal $\left(\Delta m_{31}^{2}>0\right)$ or inverted $\left(\Delta m_{31}^{2}<0\right)$. Matter effects have been checked to be negligible for $\Delta m_{41}^{2}>1 \mathrm{eV}^{2}$. 
Observed neutrino oscillation anomalies [23], if interpreted in terms of one additional sterile neutrino, suggest $\left|\Delta m_{41}^{2}\right|$ values at the $\mathrm{eV}^{2}$ scale (the so-called $3+1$ model). In the following, unless stated otherwise, the analysis will be restricted only to positive $\Delta m_{41}^{2}$ values, since negative values are disfavoured by results on the sum of neutrino masses from cosmological surveys [13]. For $\Delta m_{41}^{2}>1 \mathrm{eV}^{2}$, at the concerned domain of $L / E$ and taking into account the finite energy resolution, $\sin 2 \Delta_{41}$ and $\sin ^{2} \Delta_{41}$ average to 0 and $\frac{1}{2}$, respectively. The oscillation probability $P(E)$ can thus be approximated to [23]:

$$
\begin{aligned}
P(E)= & C^{2} \sin ^{2} \Delta_{31}+\frac{1}{2} \sin ^{2} 2 \theta_{\mu \tau} \\
& +C \sin 2 \theta_{\mu \tau} \cos \phi_{\mu \tau} \sin ^{2} \Delta_{31} \\
& +\frac{1}{2} C \sin 2 \theta_{\mu \tau} \sin \phi_{\mu \tau} \sin 2 \Delta_{31} .
\end{aligned}
$$

In order to obtain an upper limit on $\sin ^{2} 2 \theta_{\mu \tau}$ at high values of $\Delta m_{41}^{2}$, the likelihood is defined as $L\left(\phi_{\mu \tau}, \sin ^{2} 2 \theta_{\mu \tau}, C^{2}\right)=e^{-\mu} \mu^{n} / n$ !, where $n=4$ is the number of $\nu_{\tau}$ candidate events and $\mu$ is the expected number of events, $\mu=n_{b}+A \int \phi(E) P(E) \sigma(E) \epsilon(E) d E$. $n_{b}=0.23$ is the expected number of background events [7], $\phi(E)$ is the $\nu_{\mu}$ flux shown in figure $1, P(E)$ is the oscillation probability given by equations (4.1) or $(4.2), \sigma(E)$ is the $\nu_{\tau}$ CC interaction cross section, $\epsilon(E)$ is the $\tau$ detection efficiency and $A$ is a normalisation factor proportional to the fraction of the analysed sample and to the target mass.

The analysis presented here is based on the asymptotic $\chi^{2}$ distribution of the $\log$ likelihood ratio test statistics: $q=-2 \ln \left(\widetilde{L}\left(\phi_{\mu \tau}, \sin ^{2} 2 \theta_{\mu \tau}\right) / L_{0}\right)$, where $L_{0}=e^{-n} n^{n} / n$ ! and $\widetilde{L}\left(\phi_{\mu \tau}, \sin ^{2} 2 \theta_{\mu \tau}\right)$ is the profile likelihood obtained by maximising $L\left(\phi_{\mu \tau}, \sin ^{2} 2 \theta_{\mu \tau}, C^{2}\right)$ over $C^{2}$. By definition, $C^{2}$ ranges between 0 and 1 , but for any pair of values of $\sin ^{2} 2 \theta_{\mu \tau}$ and $\phi_{\mu \tau}$, it is limited by the unitarity of the mixing matrix; the likelihood is maximised accordingly. The value of $\left|\Delta m_{31}^{2}\right|$ has been fixed to $2.43 \times 10^{-3} \mathrm{eV}^{2}$ for the normal hierarchy and to $2.38 \times 10^{-3} \mathrm{eV}^{2}$ for the inverted hierarchy of the three standard neutrinos [2].

In figure 2(a) the 90\% CL exclusion limits are presented for both normal and inverted mass hierarchies in the parameter space of $\phi_{\mu \tau} \mathrm{vs} \sin ^{2} 2 \theta_{\mu \tau}$. The edge of the excluded region ranges from 0.088 to 0.136 in $\sin ^{2} 2 \theta_{\mu \tau}$ for both mass hierarchies of the three standard neutrinos. For any fixed value of $\phi_{\mu \tau}, q$ is distributed according to a $\chi^{2}$ statistics with one degree of freedom. Profiling the likelihood also over $\phi_{\mu \tau}$, as shown in figure 2(b), an upper limit of 0.116 is obtained at $90 \%$ CL on $\sin ^{2} 2 \theta_{\mu \tau}$, almost independently of the hierarchy of the three standard neutrino masses. A negligible difference arises from the different $\left|\Delta m_{31}^{2}\right|$ value used in the analysis. The $\sin ^{2} 2 \theta_{\mu \tau}$ upper limit is affected by a $20 \%$ systematic error from the uncertainties on the $\tau$ detection efficiency and $\nu_{\tau}$ interaction cross section.

Given the definition of $\sin ^{2} 2 \theta_{\mu \tau}$ in terms of $U_{\mu 4}$ and $U_{\tau 4}$, it is possible to translate the upper limit on $\sin ^{2} 2 \theta_{\mu \tau}$ into an exclusion curve in the $\left|U_{\mu 4}\right|^{2}$ vs $\left|U_{\tau 4}\right|^{2}$ plane, as shown in figure 3 together with the unitarity bound $\left(\left|U_{\mu 4}\right|^{2}+\left|U_{\tau 4}\right|^{2} \leq 1\right)$.

A review of experimental neutrino oscillation results, interpreted in terms of $\left|U_{\mu 4}\right|^{2}$ and $\left|U_{\tau 4}\right|^{2}$ in the range $0.1<\Delta m_{41}^{2}<10 \mathrm{eV}^{2}$ can be found in [23]; in addition, the SuperKamiokande collaboration recently published new limits based on the analysis of atmospheric neutrinos [24]. $\left|U_{\mu 4}\right|^{2}$ is constrained by muon (anti-)neutrino disappearance experi- 

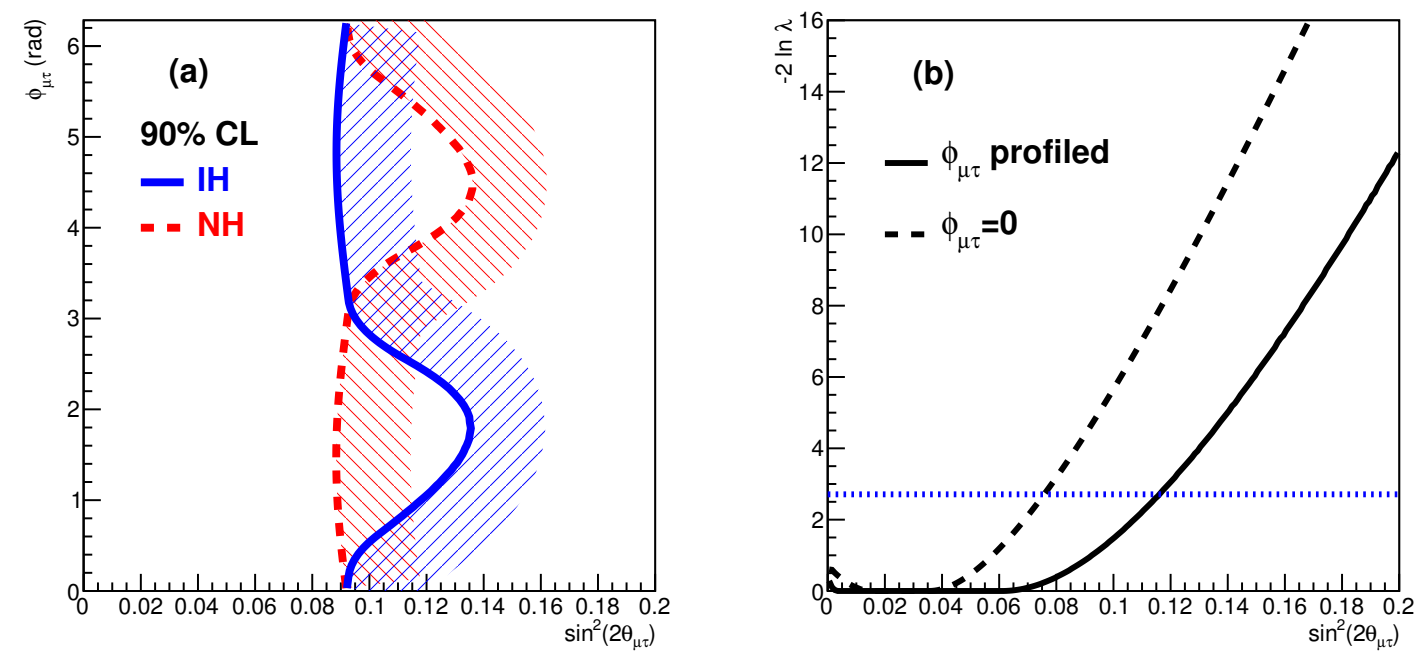

Figure 2. (a) $90 \% \mathrm{CL}$ exclusion limits in the $\phi_{\mu \tau}$ vs $\sin ^{2} 2 \theta_{\mu \tau}$ parameter space for normal (NH, dashed red) and inverted (IH, solid blue) hierarchies assuming $\Delta m_{41}^{2}>1 \mathrm{eV}^{2}$. Bands are drawn to indicate the excluded regions. (b) Log likelihood ratio as a function of $\sin ^{2} 2 \theta_{\mu \tau}$ for $\phi_{\mu \tau}=0$ (dashed line) and for the profile likelihood (continuous line).

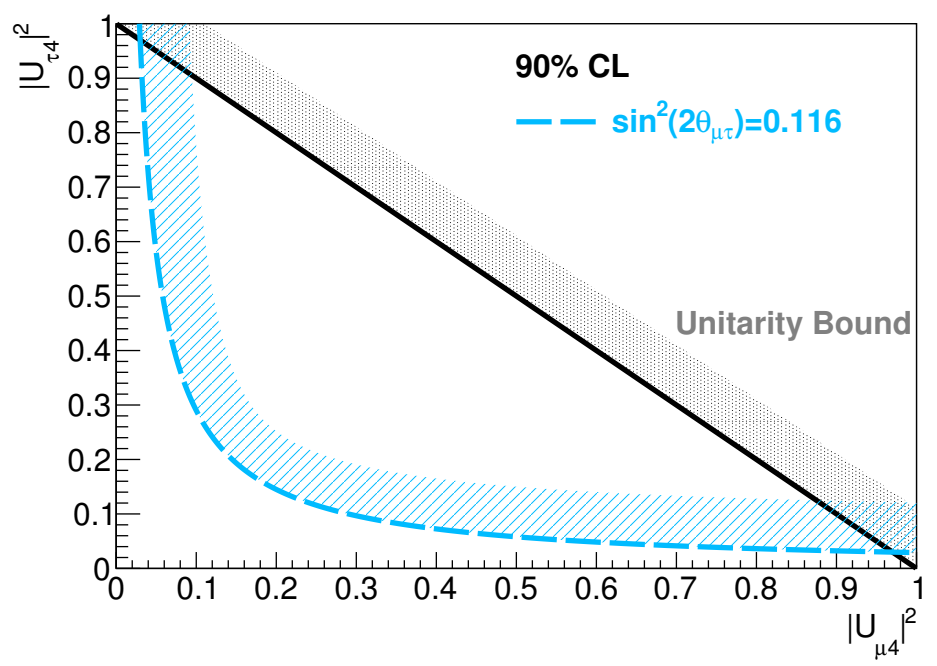

Figure 3. $90 \%$ CL exclusion limits (blue line) in the $\left|U_{\tau 4}\right|^{2}$ vs $\left|U_{\mu 4}\right|^{2}$ plane assuming $\Delta m_{41}^{2}>1 \mathrm{eV}^{2}$. The unitarity bound (grey line) is also shown. Bands are drawn to indicate the excluded regions. 


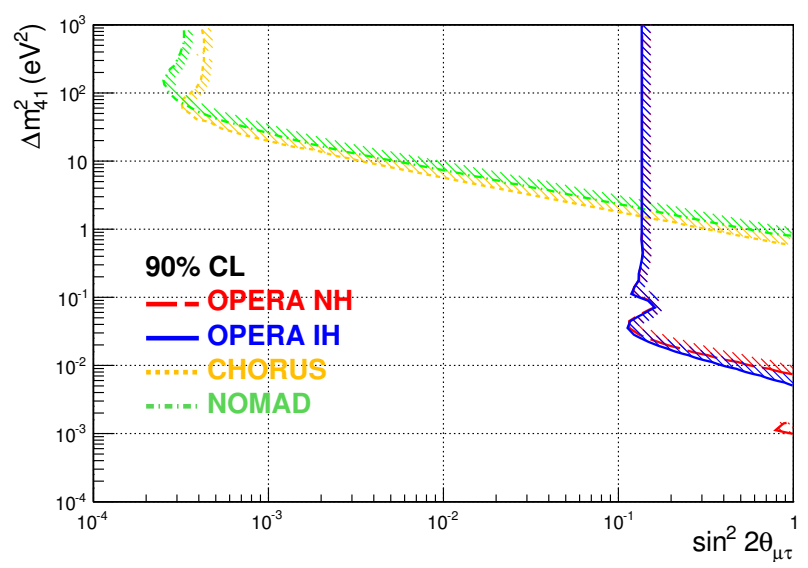

Figure 4. OPERA $90 \%$ CL exclusion limits in the $\Delta m_{41}^{2}$ vs $\sin ^{2} 2 \theta_{\mu \tau}$ parameter space for the normal ( $\mathrm{NH}$, dashed red) and inverted ( $\mathrm{IH}$, solid blue) hierarchy of the three standard neutrino masses. The exclusion plots by NOMAD [14] and CHORUS [15] are also shown. Bands are drawn to indicate the excluded regions.

ments: CDHS [25], CCFR [26], MiniBooNE+SciBooNE [27] and MINOS [28], as well as by Super-Kamiokande [24]. Limits on $\left|U_{\tau 4}\right|^{2}$ have been derived from the MINOS results on the neutral current interaction rate comparison between the near and the far detector [29] and more recently by the Super-Kamiokande analysis on matter effects for up-going atmospheric neutrinos [24]. As a consequence, for $\Delta m_{41}^{2}>0.1 \mathrm{eV}^{2}$, the $90 \%$ CL limits are $\mathcal{O}\left(10^{-2}\right)$ on $\left|U_{\mu 4}\right|^{2}$ and 0.18 on $\left|U_{\tau 4}\right|^{2}$, the latter being dominated by the Super-Kamiokande results.

To extend the search for a possible fourth sterile neutrino down to small $\Delta m_{41}^{2}$ values, the likelihood has been computed using the GLoBES software [30, 31], which takes into account the non-zero $\Delta m_{21}^{2}$ value and also matter effects, the Earth density being approximated by a constant value estimated with the PREM $[32,33]$ onion shell model. The likelihood has been profiled also on the $\Delta m_{31}^{2}$ value. More details on the analysis are available in [34]. In figure 4 the $90 \% \mathrm{CL}$ exclusion plot is reported in the $\Delta m_{41}^{2}$ vs $\sin ^{2} 2 \theta_{\mu \tau}$ parameter space. The most stringent limits of direct searches for $\nu_{\mu} \rightarrow \nu_{\tau}$ oscillations at short-baselines obtained by the NOMAD [14] and CHORUS [15] experiments are also shown. Our analysis stretches the limits on $\Delta m_{41}^{2}$ down to $10^{-2} \mathrm{eV}^{2}$, extending the values explored with the $\tau$ appearance searches by about two orders of magnitude at large mixing, for $\sin ^{2} 2 \theta_{\mu \tau} \gtrsim 0.5$. For maximal mixing, the $90 \%$ CL excluded region extends down to $\Delta m_{41}^{2}=7.4(5.2) \times 10^{-3} \mathrm{eV}^{2}$ for normal (inverted) hierarchy of the three standard neutrino masses, with a $10 \%$ systematic error deriving from the uncertainties on the $\tau$ detection efficiency and $\nu_{\tau}$ interaction cross section.

A narrow region is excluded at $90 \% \mathrm{CL}$ at $\Delta m_{41}^{2} \approx 10^{-3} \mathrm{eV}^{2}$ for the normal hierarchy of the three standard neutrinos. It arises from a suppression of the $\nu_{\mu} \rightarrow \nu_{\tau}$ oscillation probability due to the presence of the sterile neutrino. Instead, the oscillation probability is enhanced at full mixing and high $\Delta m_{41}^{2}$ values. For a number of $\tau$ neutrino candidates equal to the expectation in the three neutrino framework, the excluded region at $\Delta m_{41}^{2} \approx$ $10^{-3} \mathrm{eV}^{2}$ would disappear. 
The analysis was performed assuming $\Delta m_{41}^{2}>0$. Since present limits on the sum of neutrino masses from cosmological surveys do not exclude small negative values for $\Delta m_{41}^{2}$, the analysis was repeated following this assumption. The exclusion plots obtained in this way are similar to those of figure 4 , but with hierarchies exchanged. It is worth underlining that the results obtained in the $3+1$ model, shown in figures 2 and 3 , are independent of the sign of $\Delta m_{41}^{2}$, as is the probability in equation (4.2).

Assuming $C P$ conservation, that implies $\sin \phi_{\mu \tau}=0$, and neglecting terms in $\sin ^{2} \Delta_{31}$ in equation (4.1), of the order of $10^{-2}$ at CNGS energies $E_{C N G S}$, the oscillation probability at high values of $\Delta m_{41}^{2}$ approximates to that of a two-flavour model parametrised in terms of two effective mixing parameters, $\theta_{\text {eff }}$ and $\Delta m_{\text {eff }}^{2}$ :

$$
\lim _{E \rightarrow E_{C N G S}} P(E \mid C P \text { conservation }) \approx \sin ^{2} 2 \theta_{\text {eff }} \cdot \sin ^{2}\left(1.27 \Delta m_{\text {eff }}^{2} L / E\right)
$$

In this framework, with 4 observed events and $2.53 \pm 0.46$ events expected from the normal hierarchy of standard oscillations, including background, the upper limit on the number of additional $\nu_{\tau}$ events, evaluated in the Feldman-Cousins approach [35], is 6.4 at 90\% CL. The upper limit on $\sin ^{2} 2 \theta_{\text {eff }}$ is 0.069 , to be compared with 0.076 for $\sin ^{2} 2 \theta_{\mu \tau}$ at $\phi_{\mu \tau}=0$ (see figure 2(b)).

\section{Conclusions}

The OPERA experiment was designed to observe $\nu_{\mu} \rightarrow \nu_{\tau}$ oscillations through $\nu_{\tau}$ appearance at a baseline of $730 \mathrm{~km}$ in the CNGS beam. Exploiting its unique capability to identify $\tau$ neutrino interactions, OPERA has observed four $\nu_{\tau} \mathrm{CC}$ candidate interactions, consistent with the expected number of oscillation events in the standard three-neutrino framework, $2.30 \pm 0.46(2.21 \pm 0.44)$, for the normal (inverted) mass hierarchy and 0.23 \pm 0.05 background events.

In this paper we present limits on the existence of a sterile neutrino in the $3+1$ neutrino model. At high values of $\Delta m_{41}^{2}$, the measured $90 \%$ CL upper limit on the mixing term $\sin ^{2} 2 \theta_{\mu \tau}=4\left|U_{\mu 4}\right|^{2}\left|U_{\tau 4}\right|^{2}$ is 0.116 , independently of the mass hierarchy of the three standard neutrinos. The OPERA experiment extends the exclusion limits on $\Delta m_{41}^{2}$ in the $\nu_{\mu} \rightarrow \nu_{\tau}$ appearance channel down to values of $10^{-2} \mathrm{eV}^{2}$ at large mixing for $\sin ^{2} 2 \theta_{\mu \tau} \gtrsim 0.5$.

\section{Acknowledgments}

We thank CERN for the successful operation of the CNGS facility and INFN for the continuous support given to the experiment through its LNGS laboratory. We acknowledge funding from our national agencies: Fonds de la Recherche Scientique-FNRS and Institut InterUniversitaire des Sciences Nucleaires for Belgium, MoSES for Croatia, CNRS and IN2P3 for France, BMBF for Germany, INFN for Italy, JSPS (Japan Society for the Promotion of Science), MEXT (Ministry of Education, Culture, Sports, Science and Technology), QFPU (Global COE programme of Nagoya University, Quest for Fundamental Principles in the Universe supported by JSPS and MEXT) and Promotion and Mutual Aid Corporation for Private Schools of Japan for Japan, SNF, the University of Bern for Switzerland, the 
Russian Foundation for Basic Research (grant no. 19-02-00213-a, 12-02-12142 ofim), the Programs of the Presidium of the Russian Academy of Sciences Neutrino physics and Experimental and theoretical researches of fundamental interactions connected with work on the accelerator of CERN, the Programs of Support of Leading Schools (grant no. 3110.2014.2), and the Ministry of Education and Science of the Russian Federation for Russia and the National Research Foundation of Korea Grant (NRF-2013R1A1A2061654) for Korea. We are also indebted to INFN for providing fellowships and grants to non-Italian researchers. We thank the IN2P3 Computing Centre (CC-IN2P3) for providing computing resources for the analysis and hosting the central database for the OPERA experiment. We are indebted to our technical collaborators for the excellent quality of their work over many years of design, prototyping, construction and running of the detector and of its facilities. We also want to thank Carlo Giunti, Francesco Vissani and Enrico Nardi for fruitful discussions.

Open Access. This article is distributed under the terms of the Creative Commons Attribution License (CC-BY 4.0), which permits any use, distribution and reproduction in any medium, provided the original author(s) and source are credited.

\section{References}

[1] OPERA collaboration, R. Acquafredda et al., The OPERA experiment in the CERN to Gran Sasso neutrino beam, 2009 JINST 4 P04018 [INSPIRE].

[2] Particle Data Group collaboration, K.A. Olive et al., Review of particle physics, Chin. Phys. C 38 (2014) 090001 [INSPIRE].

[3] Super-Kamiokande collaboration, Y. Fukuda et al., Evidence for oscillation of atmospheric neutrinos, Phys. Rev. Lett. 81 (1998) 1562 [hep-ex/9807003] [INSPIRE].

[4] OPERA collaboration, N. Agafonova et al., Observation of a first $\nu_{\tau}$ candidate in the OPERA experiment in the CNGS beam, Phys. Lett. B 691 (2010) 138 [arXiv:1006.1623] [INSPIRE].

[5] OPERA collaboration, N. Agafonova et al., New results on $\nu_{\mu} \rightarrow \nu_{\tau}$ appearance with the OPERA experiment in the CNGS beam, JHEP 11 (2013) 036 [Erratum ibid. 04 (2014) 014] [arXiv: 1308.2553] [INSPIRE].

[6] OPERA collaboration, N. Agafonova et al., Evidence for $\nu_{\mu} \rightarrow \nu_{\tau}$ appearance in the CNGS neutrino beam with the OPERA experiment, Phys. Rev. D 89 (2014) 051102 [arXiv: 1401.2079] [INSPIRE].

[7] OPERA collaboration, N. Agafonova et al., Observation of $\tau$ neutrino appearance in the CNGS beam with the OPERA experiment, Prog. Theor. Exp. Phys. 2014 (2014) 101C01 [arXiv: 1407.3513] [INSPIRE].

[8] LSND collaboration, A. Aguilar-Arevalo et al., Evidence for neutrino oscillations from the observation of $\bar{\nu}_{e}$ appearance in a $\bar{\nu}_{\mu}$ beam, Phys. Rev. D 64 (2001) 112007 [hep-ex/0104049] [INSPIRE].

[9] MiniBoonE collaboration, A.A. Aguilar-Arevalo et al., Improved search for $\bar{\nu}_{\mu} \rightarrow \bar{\nu}_{e}$ oscillations in the MiniBooNE experiment, Phys. Rev. Lett. 110 (2013) 161801 [arXiv: 1207.4809] [INSPIRE]. 
[10] G. Mention et al., The reactor antineutrino anomaly, Phys. Rev. D 83 (2011) 073006 [arXiv:1101.2755] [INSPIRE].

[11] M.A. Acero, C. Giunti and M. Laveder, Limits on $\nu_{e}$ and $\bar{\nu}_{e}$ disappearance from Gallium and reactor experiments, Phys. Rev. D 78 (2008) 073009 [arXiv:0711.4222] [INSPIRE].

[12] C. Giunti and M. Laveder, Statistical significance of the Gallium anomaly, Phys. Rev. C 83 (2011) 065504 [arXiv: 1006.3244] [INSPIRE].

[13] Planck collaboration, P.A.R. Ade et al., Planck 2015 results. XIII. Cosmological parameters, [arXiv:1502.01589] [INSPIRE].

[14] NOMAD collaboration, P. Astier et al., Final NOMAD results on $\nu_{\mu} \rightarrow \nu_{\tau}$ and $\nu_{e} \rightarrow \nu_{\tau}$ oscillations including a new search for $\nu_{\tau}$ appearance using hadronic $\tau$ decays, Nucl. Phys. B 611 (2001) 3 [hep-ex/0106102] [INSPIRE].

[15] CHORUS collaboration, E. Eskut et al., Final results on $\nu_{\mu} \rightarrow \nu_{\tau}$ oscillation from the CHORUS experiment, Nucl. Phys. B 793 (2008) 326 [arXiv:0710.3361] [InSPIRE].

[16] N. Agafonova et al., The detection of neutrino interactions in the emulsion/lead target of the OPERA experiment, 2009 JINST 4 P06020 [arXiv:0903.2973] [INSPIRE].

[17] K. Elsener, The CERN neutrino beam to Gran Sasso (NGS): conceptual technical design, CERN-98-02, CERN, Geneva Switzerland (1998) [INFN-AE-98-05].

[18] R. Bailey et al., The CERN Neutrino beam to Gran Sasso (NGS): addendum to report no. CERN-98-02, INFN-AE-98-05, CERN-SL-99-034(DI), CERN, Geneva Switzerland (1999) [INFN-AE-99-05].

[19] CNGS neutrino flux calculations webpage, http://www.mi.infn.it/ psala/Icarus/cngs.html.

[20] OPERA collaboration, A. Anokhina et al., Emulsion sheet doublets as interface trackers for the OPERA experiment, 2008 JINST 3 P07005 [arXiv:0804.1985] [INSPIRE].

[21] OPERA collaboration, N. Agafonova et al., Procedure for short-lived particle detection in the OPERA experiment and its application to charm decays, Eur. Phys. J. C 74 (2014) 2986 [arXiv: 1404.4357] [INSPIRE].

[22] Particle Data Group collaboration, J. Beringer et al., Review of particle physics (RPP), Phys. Rev. D 86 (2012) 010001 [INSPIRE].

[23] J. Kopp, P.A.N. Machado, M. Maltoni and T. Schwetz, Sterile neutrino oscillations: the global picture, JHEP 05 (2013) 050 [arXiv: 1303.3011] [INSPIRE].

[24] Super-KamiokAnde collaboration, K. Abe et al., Limits on sterile neutrino mixing using atmospheric neutrinos in Super-Kamiokande, Phys. Rev. D 91 (2015) 052019 [arXiv: 1410.2008] [INSPIRE].

[25] F. Dydak et al., A search for $\nu_{\mu}$ oscillations in the $\Delta m^{2}$ range $0.3 \mathrm{eV}^{2}$ to $90 \mathrm{eV}^{2}$, Phys. Lett. B 134 (1984) 281 [INSPIRE].

[26] I.E. Stockdale et al., Limits on muon neutrino oscillations in the mass range $55 \mathrm{eV}^{2}<\Delta m^{2}<800 \mathrm{eV}^{2}$, Phys. Rev. Lett. 52 (1984) 1384 [InSPIRE].

[27] MiniBoone and SciBoone collaborations, G. Cheng et al., Dual baseline search for muon antineutrino disappearance at $0.1 \mathrm{eV}^{2}<\Delta m^{2}<100 \mathrm{eV}^{2}$, Phys. Rev. D 86 (2012) 052009 [arXiv:1208.0322] [INSPIRE].

[28] MINOS collaboration, P. Adamson et al., Search for sterile neutrino mixing in the MINOS long baseline experiment, Phys. Rev. D 81 (2010) 052004 [arXiv:1001.0336] [INSPIRE]. 
[29] MINOS collaboration, P. Adamson et al., Active to sterile neutrino mixing limits from neutral-current interactions in MINOS, Phys. Rev. Lett. 107 (2011) 011802 [arXiv:1104.3922] [INSPIRE].

[30] P. Huber, M. Lindner and W. Winter, Simulation of long-baseline neutrino oscillation experiments with GLoBES (General Long Baseline Experiment Simulator), Comput. Phys. Commun. 167 (2005) 195 [hep-ph/0407333] [INSPIRE].

[31] P. Huber, J. Kopp, M. Lindner, M. Rolinec and W. Winter, New features in the simulation of neutrino oscillation experiments with GLoBES 3.0: General Long Baseline Experiment Simulator, Comput. Phys. Commun. 177 (2007) 432 [hep-ph/0701187] [INSPIRE].

[32] A.M. Dziewonski and D.L. Anderson, Preliminary reference earth model, Phys. Earth Planet. Interiors 25 (1981) 297 [INSPIRE].

[33] F.D. Stacey, Physics of the earth, $2^{\text {nd }}$ ed., Wiley, U.S.A. (1977).

[34] S. Dusini et al., Search for sterile neutrino mixing in the $\nu_{\mu} \rightarrow \nu_{\tau}$ appearance channel with the OPERA detector, http://operaweb.lngs.infn.it/Opera/publicnotes/OPERA-public-note-175.pdf.

[35] G.J. Feldman and R.D. Cousins, A unified approach to the classical statistical analysis of small signals, Phys. Rev. D 57 (1998) 3873 [physics/9711021] [INSPIRE]. 


\section{The OPERA collaboration}

N. Agafonova,${ }^{a}$ A. Aleksandrov,${ }^{b}$ A. Anokhina,${ }^{c}$ S. Aoki,${ }^{d}$ A. Ariga,${ }^{e}$ T. Ariga,${ }^{e}$ D. Bender,${ }^{f}$

A. Bertolin, ${ }^{g}$ I. Bodnarchuk, ${ }^{h}$ C. Bozza, ${ }^{i}$ R. Brugnera, ${ }^{g, j}$ A. Buonaura, ${ }^{b, k}$ S. Buontempo, ${ }^{b}$

B. Büttner, ${ }^{l}$ M. Chernyavsky, ${ }^{m}$ A. Chukanov, ${ }^{h}$ L. Consiglio, ${ }^{b}$ N. D'Ambrosio, ${ }^{n}$ G. De Lellis $,{ }^{b}, k$ M. De Serio, ${ }^{o, p}$ P. Del Amo Sanchez,${ }^{q}$ A. Di Crescenzo, ${ }^{b}$ D. Di Ferdinando, ${ }^{r}$ N. Di Marco, ${ }^{n}$

S. Dmitrievski, ${ }^{h}$ M. Dracos, ${ }^{s}$ D. Duchesneau, ${ }^{q}$ S. Dusini, ${ }^{g}$ T. Dzhatdoev, ${ }^{c}$ J. Ebert,${ }^{l}$

A. Ereditato, ${ }^{e}$ R. A. Fini, ${ }^{p}$ T. Fukuda, ${ }^{t}$ G. Galati, ${ }^{b, k}$ A. Garfagnini, ${ }^{i}{ }^{i}$ J. Goldberg, ${ }^{v}$

Y. Gornushkin, ${ }^{h}$ G. Grella,${ }^{i}$ A.M. Guler,${ }^{f}$ C. Gustavino, ${ }^{w}$ C. Hagner, ${ }^{l}$ T. Hara,${ }^{d}$

A. Hollnagel,${ }^{l}$ B. Hosseini ${ }^{b, k}$ K. Ishiguro, ${ }^{x}$ K. Jakovcic,${ }^{y}$ C. Jollet,${ }^{s}$ C. Kamiscioglu, ${ }^{f}$

M. Kamiscioglu, ${ }^{f}$ J. H. Kim ${ }^{z}$ S. H. Kim, ${ }^{z, 1}$ N. Kitagawa, ${ }^{x}$ B. Klicek, ${ }^{y}$ K. Kodama, ${ }^{a a}$

M. Komatsu, ${ }^{x}$ U. Kose, ${ }^{g, 2}$ I. Kreslo, ${ }^{e}$ A. Lauria, ${ }^{b, k}$ A. Ljubicic, ${ }^{y}$ A. Longhin, ${ }^{a b}$ A. Malgin, ${ }^{a}$

M. Malenica, ${ }^{y}$ G. Mandrioli, ${ }^{r}$ T. Matsuo, ${ }^{t}$ V. Matveev,${ }^{a}$ N. Mauri, ${ }^{r, u}$ E. Medinaceli,${ }^{g, j}$

A. Meregaglia, ${ }^{s}$ S. Mikado, ${ }^{a d}$ P. Monacelli, ${ }^{w}$ M. C. Montesi, ${ }^{b, k}$ K. Morishima, ${ }^{x}$

M. T. Muciaccia,${ }^{o, p}$ N. Naganawa,${ }^{x}$ T. Naka,${ }^{x}$ M. Nakamura,${ }^{x}$ T. Nakano,${ }^{x}$ Y. Nakatsuka,${ }^{x}$

K. Niwa ${ }^{x}$ S. Ogawa, ${ }^{t}$ T. Omura ${ }^{x}$ K. Ozaki, ${ }^{d}$ A. Paoloni, ${ }^{a b, *}$ L. Paparella, ${ }^{o, p}$ B. D. Park, ${ }^{z, 3}$

I. G. Park,${ }^{z}$ L. Pasqualini, ${ }^{r, u}$ A. Pastore,${ }^{p, *}$ L. Patrizii, ${ }^{r}$ H. Pessard, ${ }^{q}$ D. Podgrudkov,${ }^{c}$

N. Polukhina, ${ }^{m}$ M. Pozzato, ${ }^{r, u}$ F. Pupilli, ${ }^{n}$ M. Roda,${ }^{g, j}$ T. Roganova,${ }^{c}$ H. Rokujo, ${ }^{x}$

G. Rosa,${ }^{w, a c}$ O. Ryazhskaya, ${ }^{a}$ O. Sato ${ }^{x}$ A. Schembri, ${ }^{n}$ I. Shakirianova, ${ }^{a}$ T. Shchedrina, ${ }^{b}$

A. Sheshukov ${ }^{h}$ H. Shibuya, ${ }^{t}$ T. Shiraishi, ${ }^{x}$ G. Shoziyoev, ${ }^{c}$ S. Simone, ${ }^{o, p}$ M. Sioli,${ }^{r, u}$

C. Sirignano, ${ }^{g, j}$ G. Sirri, ${ }^{r}$ M. Spinetti, ${ }^{a b}$ L. Stanco, ${ }^{g}$ N. Starkov, ${ }^{m}$ S. M. Stellacci, ${ }^{i}$

M. Stipcevic, ${ }^{y}$ P. Strolin,${ }^{b, k}$ S. Takahashi, ${ }^{d}$ M. Tenti, ${ }^{r}$ F. Terranova, ${ }^{a b, a e}$ V. Tioukov, ${ }^{b}$

S. Tufanli, ${ }^{e}$ P. Vilain, ${ }^{a f}$ M. Vladymyrov ${ }^{m}$ L. Votano, ${ }^{a b}$ J. L. Vuilleumier, ${ }^{e}$ G. Wilquet, ${ }^{a f}$

B. Wonsak, ${ }^{l}$ C. S. Yoon, ${ }^{z}$ S. Zemskova ${ }^{h}$

a INR - Institute for Nuclear Research of the Russian Academy of Sciences, RUS-117312 Moscow, Russia

${ }^{b}$ INFN Sezione di Napoli, 80125 Napoli, Italy

c SINP MSU - Skobeltsyn Institute of Nuclear Physics, Lomonosov Moscow State University, RUS-119991 Moscow, Russia

d Kobe University, J-657-8501 Kobe, Japan

e Albert Einstein Center for Fundamental Physics, Laboratory for High Energy Physics (LHEP), University of Bern, CH-3012 Bern, Switzerland

${ }^{f}$ METU - Middle East Technical University, TR-06531 Ankara, Turkey

g INFN Sezione di Padova, I-35131 Padova, Italy

h JINR - Joint Institute for Nuclear Research, RUS-141980 Dubna, Russia

i Dipartimento di Fisica dell'Università di Salerno and "Gruppo Collegato" INFN, I-84084 Fisciano (Salerno), Italy

j Dipartimento di Fisica e Astronomia dell'Università di Padova, I-35131 Padova, Italy

${ }^{k}$ Dipartimento di Fisica dell'Università Federico II di Napoli, I-80125 Napoli, Italy

$l$ Hamburg University, D-22761 Hamburg, Germany

$m$ LPI - Lebedev Physical Institute of the Russian Academy of Sciences, RUS-119991 Moscow, Russia

$n$ INFN - Laboratori Nazionali del Gran Sasso, I-67010 Assergi (L'Aquila), Italy

- Dipartimento di Fisica dell'Università di Bari, I-70126 Bari, Italy

p INFN Sezione di Bari, I-70126 Bari, Italy

q LAPP, Université Savoie Mont Blanc, CNRS/IN2P3, F-74941 Annecy-le-Vieux, France

${ }^{r}$ INFN Sezione di Bologna, I-40127 Bologna, Italy

\footnotetext{
${ }^{1}$ Now at Kyungpook National University, Daegu, Korea.

${ }^{2}$ Now at CERN, Geneva, Switzerland.

${ }^{3}$ Now at Samsung Changwon Hospital, SKKU, Changwon, Korea.
} 
s IPHC, Université de Strasbourg, CNRS/IN2P3, F-67037 Strasbourg, France

$t$ Toho University, J-274-8510 Funabashi, Japan

u Dipartimento di Fisica e Astronomia dell'Università di Bologna, I-40127 Bologna, Italy

$v$ Department of Physics, Technion, IL-32000 Haifa, Israel

w INFN Sezione di Roma, I-00185 Roma, Italy

$x$ Nagoya University, J-464-8602 Nagoya, Japan

y IRB - Rudjer Boskovic Institute, HR-10002 Zagreb, Croatia

$z$ Gyeongsang National University, 900 Gazwa-dong, Jinju 660-701, Korea

aa Aichi University of Education, J-448-8542 Kariya (Aichi-Ken), Japan

ab INFN - Laboratori Nazionali di Frascati dell'INFN, I-00044 Frascati (Roma), Italy

ac Dipartimento di Fisica dell'Università di Roma "La Sapienza", I-00185 Roma, Italy

ad Nihon University, J-275-8576 Narashino, Chiba, Japan

ae Dipartimento di Fisica dell'Università di Milano-Bicocca, I-20126 Milano, Italy

af IIHE, Université Libre de Bruxelles, B-1050 Brussels, Belgium 